\title{
Mixed convection boundary layer flow past a horizontal circular cylinder in viscoelastic nanofluid with constant wall temperature
}

\author{
Rahimah Mahat ${ }^{a, b}$, Noraihan Afiqah Rawi ${ }^{b}$, Abdul Rahman Mohd Kasim ${ }^{c}$, Sharidan Shafie ${ }^{b,{ }^{*}}$ \\ a Universiti Kuala Lumpur Malaysian Institute Technology of Industrial Technology, Persiaran Sinaran Ilmu, Johor Bahru, Johor, Malaysia \\ ${ }^{b}$ Department of Mathematics, Faculty of Science, Universiti Teknologi Malaysia, 81310 UTM Johor Bahru, Johor, Malaysia \\ c Universiti Malaysia Pahang, Lebuhraya Tun Razak, 26300 Gambang, Kuantan, Pahang, Malaysia
}

* Corresponding sharidan@utm.my

\section{Article history}

Received 24 October 2017

Accepted 8 November 2017

\begin{abstract}
The steady of two-dimensional convection boundary layer flow of viscoelastic nanofluid over a circular cylinder with constant wall temperature is investigated in this paper. Carboxymethyl cellulose solution-water (CMC-water) is chosen as the base fluid and copper as a nanoparticle with the Prandtl number $\operatorname{Pr}=6.2$. The governing boundary layer partial differential equations are transformed into dimensionless forms. Then the obtained equations are solved numerically by using the Keller-Box method. This paper focus on the effect of selected parameter on the flow and heat transfer characteristics and be presented in graphs. The results show that, the velocity profiles are increased while the temperature profiles are decreased by increasing the values of nanoparticles volume fraction and viscoelastic parameter, respectively. Also, the values of reduced skin friction are increased by increasing mixed convection parameter, but the values of heat transfer coefficient produce an opposite behavior with an increasing in mixed convection parameter.
\end{abstract}

Keywords: Viscoelastic, nanofluid, mixed convection, horizontal circular cylinder, constant wall temperature

(c) 2017 Penerbit UTM Press. All rights reserved

\section{INTRODUCTION}

The concept of nanofluid was first manifested by series of research at Argonne National Laboratory and Choi [1] was the first to call the fluids with particles of nanometer dimension suspended in them as "Nano-fluids" which has gained popularity. Nanoparticles used in nanofluids can be classified by materials. The nanoparticles are consisting of nano sized metals, oxides and carbon nanotubes. Recently, study of nanofluids has become most popular among researchers' due to its various applications in many industries, engineering and medical sciences as well. Basically, this kind of fluid have extremely high thermal conductivities compared to the conventional liquids, then nanofluids have been proposed as a route for surpassing the performance of heat transfer liquids. Many researchers had involved in the study of boundary layer flow problem in nanofluids such as, Nield and Kuznetsov [2], Khan and Pop [3], Ahmad and Pop[4], Chamkha et al. [5] ,Qasim et al. [6], Zaib et al. [7] and Noghrehabadi et al. [8]. Recently, Sabir et al. [9] investigated mixed convection flow of viscoelastic nanofluid by a cylinder with variable thermal conductivity and heat source/sink. In reality, most fluids are non-Newtonian. Non-Newtonian fluid means that their viscosity is dependent on shear rate (thickening) or the deformation history. Nowadays, the non-Newtonian nanofluids have received much considerable interest and concern by the researchers' due to the potential of nanofluids applications in many types of industry. Rawi et al. [10], Mabood et al. [11], Sarkar et al. [12], and Bouchoucha and Bessaih [13] have been considered some interesting studies involving the non-Newtonian nanofluids with various conditions and geometries have been considered. Motivated by the above-mentioned studies, this paper considers the steady of two-dimensional mixed convection boundary layer flow past a horizontal circular cylinder in viscoelastic nanofluid with constant wall temperature.The Tiwari and Das model
[14] was used in this study. To the best of our knowledge, this problem has never been considered before and the results reported here are new. The influence of relevant parameters on the dimensionless fluid velocity, the temperature, the nanoparticle volume fraction, the rate of heat transfer, and the rate of skin friction coefficients are investigated and shown graphically and discussed.

\section{MATHEMATICAL FORMULATION}

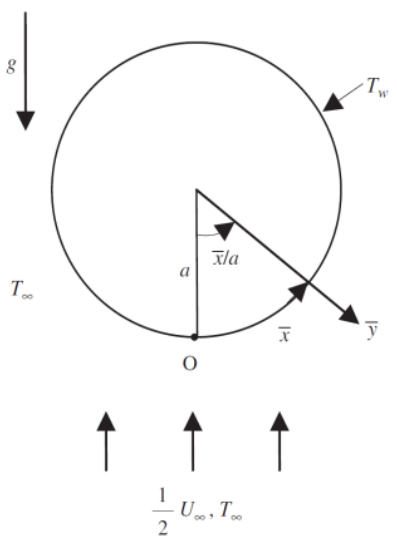

Fig.1 Physical model and coordinate system.

In this paper, the steady mixed convection boundary layer flow past an isothermal horizontal circular cylinder of radius a placed in a viscoelastic nanofluid is studied. Fig. 1 illustrates the geometry of the problem and the corresponding coordinate system. It is assumed that 
the constant temperature of the surface of the cylinder is $T_{w}$, and that of the ambient fluid is $T_{\infty}$, where $T_{w}>T_{\infty}$ corresponds to a heated cylinder (assisting flow) and $T_{w}<T_{\infty}$ corresponds to a cooled cylinder (opposing flow), respectively. It is also assumed that the viscous dissipation is neglected. Further, following Merkin [15], we assume that the velocity of the freestream is $(1 / 2) U_{\infty}$. Under these assumptions along with the Boussinesq approximation, the boundary layer equations can be written as follows, Merkin [15], Nazar [16];

$\frac{\partial \bar{u}}{\partial \bar{x}}+\frac{\partial \bar{v}}{\partial \bar{y}}=0$,

$\rho_{n f}\left(\bar{u} \frac{\partial \bar{u}}{\partial \bar{x}}+\bar{v} \frac{\partial \bar{u}}{\partial \bar{y}}\right)=\rho_{n f} \bar{u}_{e} \frac{\partial \bar{u}_{e}}{\partial \bar{x}}+\mu_{n f} \frac{\partial^{2} \bar{u}}{\partial \bar{y}^{2}}$

$+k_{0}\left[\frac{\partial}{\partial \bar{x}}\left(\bar{u} \frac{\partial^{2} \bar{u}}{\partial \bar{y}^{2}}+\bar{v} \frac{\partial^{3} \bar{u}}{\partial \bar{y}^{3}}-\frac{\partial \bar{u}}{\partial \bar{y}} \frac{\partial^{2} \bar{u}}{\partial \bar{x} \partial \bar{y}}\right)\right]+g(\rho \beta)_{n f}\left(T-T_{\infty}\right) \sin \left(\frac{\bar{x}}{a}\right)$,

$\left(\rho C_{p}\right)_{n f}\left[\bar{u} \frac{\partial T}{\partial \bar{x}}+\bar{v} \frac{\partial T}{\partial \bar{y}}\right]=k_{n f} \frac{\partial^{2} T}{\partial \bar{y}^{2}}$

subjected to the boundary conditions:

$$
\begin{array}{lll}
\bar{u}=0, \bar{v}=0, T=T_{w} & \text { at } & \bar{y}=0, \bar{x} \geq 0, \\
\bar{u}=\bar{u}_{e}(\bar{x}), \frac{\partial \bar{u}}{\partial \bar{y}}=0, T=T_{\infty} & \text { as } & \bar{y} \rightarrow \infty, \bar{x} \geq 0,
\end{array}
$$

where $\bar{x}$ and $\bar{y}$ are the Cartesian coordinates along the surface of the cylinder. The value is starting from the lower stagnation point of the cylinder. While $\bar{y}$ is the coordinate measured normal to the surface of the cylinder, $\bar{u}$ and $\bar{v}$ are the velocity components, $\bar{u}_{e}(\bar{x})$ is the velocity outside the boundary layer, $T$ is the temperature of selected fluid, $k_{0}(\geq 0)$ is the constant of the viscoelastic material with $k_{0}=0, \rho_{n f}$ and $\mu_{n f}$ are the density and dynamic viscosity of nanofluid, $(\beta)_{n f}$ is the thermal expansion of nanofluid, $k_{n f}$ is the effective thermal conductivity of the nanofluid and $\left(\rho C_{p}\right)_{n f}$ is the heat capacitance of nanofluid. These nanofluid constants are defined by,

$$
\begin{aligned}
& \left(\rho C_{p}\right)_{n f}=(1-\phi)\left(\rho C_{p}\right)_{f}+\phi\left(\rho C_{p}\right)_{s}, \\
& (\rho \beta)_{n f}=(1-\phi)(\rho \beta)_{f}+\phi(\rho \beta)_{s}, \\
& \mu_{n f}=\frac{\mu_{f}}{(1-\phi)^{2.5}}, \rho_{n f}=(1-\phi) \rho_{f}+\phi \rho_{s}, \\
& k_{n f}=k_{f} \frac{\left(k_{s}+2 k_{f}\right)-2 \phi\left(k_{f}-k_{s}\right)}{\left(k_{s}+2 k_{f}\right)+\phi\left(k_{f}-k_{s}\right)} .
\end{aligned}
$$

where $\phi$ is the nanoparticle volume fraction of the nanofluid. The thermophysical properties of nanoparticles and base fluid is given in Table 1 [17].

Table 1 Thermophysical properties of nanoparticles and base fluid.

Physical Properties

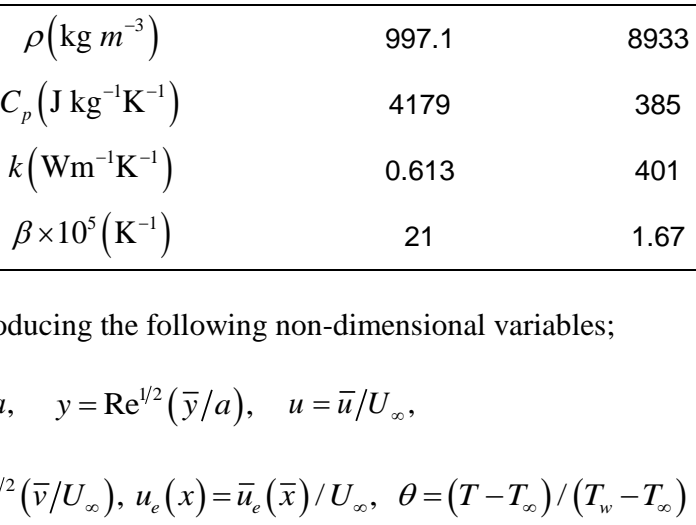

where $\operatorname{Re}=U_{\infty} a / v$ is the Reynolds number. By substitution equation (6) into equations (1)-(3), the dimensionless equations becomes,

$\frac{\partial u}{\partial x}+\frac{\partial v}{\partial y}=0$

$\left[(1-\phi)+\phi \frac{\rho_{s}}{\rho_{f}}\right]\left[u \frac{\partial u}{\partial x}+v \frac{\partial u}{\partial y}\right]=\left[(1-\phi)+\phi \frac{\rho_{s}}{\rho_{f}}\right] u_{e} \frac{\partial u_{e}}{\partial x}+\frac{1}{(1+\phi)^{2.5}} \frac{\partial^{2} u}{\partial y^{2}}$

$+K\left[\frac{\partial}{\partial x}\left(u \frac{\partial^{2} u}{\partial y^{2}}\right)+v \frac{\partial^{3} u}{\partial y^{3}}-\frac{\partial u}{\partial y} \frac{\partial^{2} u}{\partial x \partial y}\right]+\left[(1-\phi)+\phi \frac{(\rho \beta)_{s}}{(\rho \beta)_{f}}\right] \lambda \theta \sin (x)$,

$\left[(1-\phi)+\phi \frac{\left(\rho C_{p}\right)_{s}}{\left(\rho C_{p}\right)_{f}}\right]\left[u \frac{\partial \theta}{\partial x}+v \frac{\partial \theta}{\partial y}\right]=\frac{k_{n f}}{k_{f}} \frac{1}{\operatorname{Pr}} \frac{\partial^{2} \theta}{\partial y^{2}}$,

with the boundary conditions,

$\begin{array}{llll}u=0, \quad v=0, \quad \theta=1 \quad \text { at } & y=0, x \geq 0, \\ u=u_{e}(x), \frac{\partial u}{\partial y}=0, \quad \theta=0 \quad \text { as } & y \rightarrow \infty, x \geq 0,\end{array}$

where $\operatorname{Pr}$ is the Prandtl number, $K$ known as dimensionless viscoelastic parameter and is known as constant mixed convection parameter, that are defined by,

$$
K=\frac{k_{0} U_{\infty}}{a \rho v}, \quad \lambda=\frac{G r}{\operatorname{Re}^{2}},
$$

where $G r=g \beta\left(T_{w}-T_{\infty}\right) a^{3} / v^{2}$ is also known as a Grashof number. $\lambda>0$ is correlate to an auxiliary flow while $\lambda<0$ is correlate to the opposing flow. But $\lambda=0$ is correlate to the forced convection flow, respectively. In this problem, if $K=0$ it is referring to the case of viscous (Newtonian) fluids.

Further, we introduce now the stream function defined as usual as;

$$
\psi=x F(x, y), \quad \theta=\theta(x, y),
$$

where $\psi$ is the stream function that define as,

$$
u=\frac{\partial \psi}{\partial y}, \quad v=\frac{\partial \psi}{\partial x},
$$


Using (12) and (13) into equations (8) and (9) to obtain,

$$
\begin{aligned}
& {\left[(1-\phi)+\phi \frac{\rho_{s}}{\rho_{f}}\right]\left[\left(\frac{\partial F}{\partial y}\right)^{2}+x \frac{\partial F}{\partial y}\left(\frac{\partial^{2} F}{\partial x \partial y}\right)-x \frac{\partial F}{\partial x} \frac{\partial^{2} F}{\partial y^{2}}-F \frac{\partial^{2} F}{\partial y^{2}}\right]} \\
& =\left[(1-\phi)+\phi \frac{\rho_{s}}{\rho_{f}}\right] \frac{\sin x \cos x}{x}+\frac{1}{(1+\phi)^{2.5}} \frac{\partial^{3} F}{\partial y^{3}} \\
& +\left[(1-\phi)+\phi \frac{(\rho \beta)_{s}}{(\rho \beta)_{f}}\right] \lambda \theta \frac{\sin x}{x} \\
& +K\left[\begin{array}{l}
2 \frac{\partial F}{\partial y} \frac{\partial^{3} F}{\partial y^{3}}-F \frac{\partial^{4} F}{\partial y^{4}}-\left(\frac{\partial^{2} F}{\partial y^{2}}\right)^{2} \\
+x\left(\frac{\partial^{2} F}{\partial x \partial y} \frac{\partial^{3} F}{\partial y^{3}}-\frac{\partial F}{\partial x} \frac{\partial^{4} F}{\partial y^{4}}+\frac{\partial F}{\partial y} \frac{\partial^{4} F}{\partial x \partial y^{3}}-\frac{\partial^{2} F}{\partial y^{2}} \frac{\partial^{3} F}{\partial x \partial y^{2}}\right)
\end{array}\right], \\
& {\left[(1-\phi)+\phi \frac{\left(\rho C_{p}\right)_{s}}{\left(\rho C_{p}\right)_{f}}\right]\left[x \frac{\partial F}{\partial y} \frac{\partial \theta}{\partial x}-x \frac{\partial F}{\partial x} \frac{\partial \theta}{\partial y}-F \frac{\partial \theta}{\partial y}\right]=\frac{k_{n f}}{k_{f}} \frac{1}{\operatorname{Pr}} \frac{\partial^{2} \theta}{\partial y^{2}},}
\end{aligned}
$$

which are subject to the following boundary conditions;

$$
\begin{array}{ll}
F=0, \quad \frac{\partial F}{\partial y}=0, \theta=1, & \text { at } y=0, x \geq 0, \\
\frac{\partial F}{\partial y}=\frac{\sin x}{x}, \frac{\partial^{2} F}{\partial y^{2}}=0, \theta=0, & \text { as } y \rightarrow \infty, x \geq 0 .
\end{array}
$$

The physical quantities interest of this problem is the wall temperature distribution $\theta_{w}(x)$ and the skin friction coefficient $C_{f}$, that are defined as,

$$
C_{f}=\operatorname{Re}^{1 / 2} \frac{\tau_{w}}{\rho U_{\infty}^{2}}, \quad \theta_{w}=\operatorname{Re}^{-1 / 2} \frac{a q_{w}}{k\left(T_{w}-T_{\infty}\right)},
$$

where $\tau_{w}$ is the shear stress or skin friction while $q_{w}$ is the heat flux from the surface of cylinder [1], that are given by

$$
\begin{gathered}
\tau_{w}=\mu\left(\frac{\partial \bar{u}}{\partial \bar{y}}\right)_{\bar{y}=0}+k_{0}\left(\bar{u} \frac{\partial^{2} \bar{u}}{\partial \bar{x} \partial \bar{y}}+\bar{v} \frac{\partial^{2} \bar{u}}{\partial \bar{y}}+2 \frac{\partial \bar{u}}{\partial \bar{x}} \frac{\partial \bar{u}}{\partial \bar{y}}\right)_{\bar{y}=0}, \\
q_{w}=-k\left(\frac{\partial T}{\partial \bar{y}}\right)_{y=0} .
\end{gathered}
$$

and it is known that $\mu$ is for dynamic viscosity while $k$ is for thermal conductivity. By substitution of (6), (17) and (18), obtained

$$
C_{f}=x\left(\frac{\partial^{2} F}{\partial y^{2}}\right)_{y=0}, \quad \theta(x, 0)=\theta_{w}(x) .
$$

When $x \approx 0$, in the case of lower stagnation point, introduced the similarity variable $x f(\eta), \theta=\theta(\eta)$ and $\eta$ as per below. Thus, it can be shown that equations (7)-(9) diminish to the ordinary differential equations as:

$$
\frac{1}{(1+\phi)^{2.5}} f^{\prime \prime \prime}-\left[(1-\phi)+\phi \frac{\rho_{s}}{\rho_{f}}\right]\left[f^{\prime 2}-f f^{\prime \prime}\right]
$$

$$
\begin{gathered}
+K\left(2 f^{\prime \prime \prime \prime}-f f^{i v}-f^{\prime 2}\right)+\left[(1-\phi)+\phi \frac{(\rho \beta)_{s}}{(\rho \beta)_{f}}\right] \lambda \theta=0, \\
\frac{k_{n f}}{k_{f}} \frac{1}{\operatorname{Pr}} \theta^{\prime}+\left[(1-\phi)+\phi \frac{\left(\rho C_{p}\right)_{s}}{\left(\rho C_{p}\right)_{f}}\right] f \theta^{\prime}=0,
\end{gathered}
$$

and the boundary condition (9) as

$$
\begin{array}{lll}
f(0)=0, & f^{\prime}(0)=0, & \theta(0)=1, \\
f^{\prime}(\infty)=1, & f^{\prime \prime}(\infty)=0, & \theta(\infty)=0,
\end{array}
$$

In this case, obtained

$$
C_{f}=x f^{\prime \prime}(0), \quad \theta_{w}(0)=\theta(0) .
$$

The present study in equations (20)-(21) reduces to that of Nazar et al. [16] and Anwar et al. [18] for a viscoelastic fluid when $\phi=0$ (regular fluid).

\section{RESULTS AND DISCUSSION}

Both equations (14-15) with the boundary conditions (16), and the ordinary differential equations (20-21) with the boundary conditions (22) that were solved numerically using the Keller-box method. The effect of different values of the previous parameters on the velocity profiles, temperature profiles, reduced skin friction and heat transfer coefficients are reviewed in particularly.

Figures 2 and 3 show the comparison of the present results for the skin friction coefficient $C_{f}$ and the heat transfer from the cylinder $\theta_{w}$ with Nazar et al. [16] for a Newtonian fluid $(\mathrm{K}=0)$ with no nanofluid effects and $\operatorname{Pr}=1$. It shows that the numerical solutions obtained by the present authors are in good agreement with Nazar et al. [16]. Thus, we confident that the present results are very accurate.

Figures 4 and 5 show the local skin friction $C_{f}$ and local heat transfer $\theta_{w}$ for $\mathrm{K}=1, \operatorname{Pr}=6.2$ and various values of $\lambda$ with the presence of nanoparticle volume fraction when $\phi=0.05$. From the graph, it shows that by the presence of nanoparticle volume fraction $\phi$, the value of local skin friction is increased by increasing mixed convection parameter. The results show the same behavior for heat transfer coefficient where the value of $\theta_{w}$ is increased by increasing the values of $\lambda$

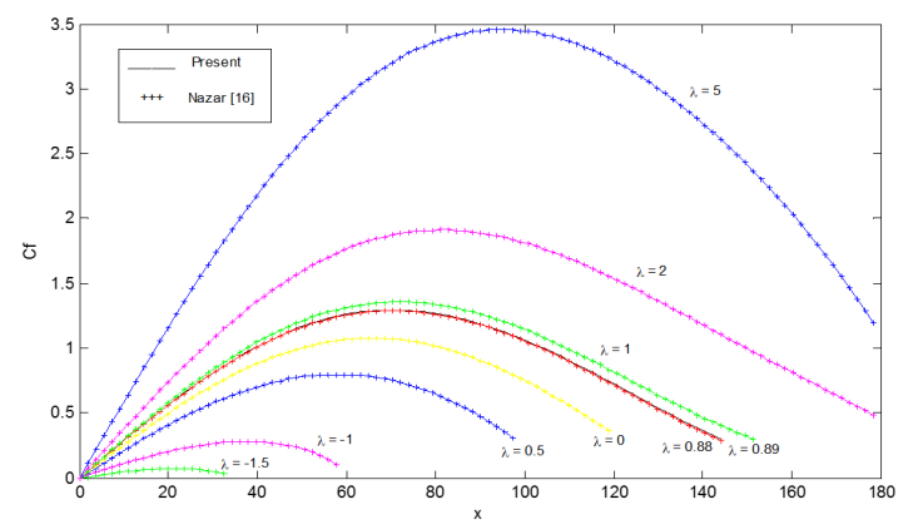

Fig. 2 Comparison of the local skin friction $C_{f}$ for $\mathrm{K}=0$ (Newtonian fluid), $\operatorname{Pr}=1, \phi=0$ and various of $\lambda$ 


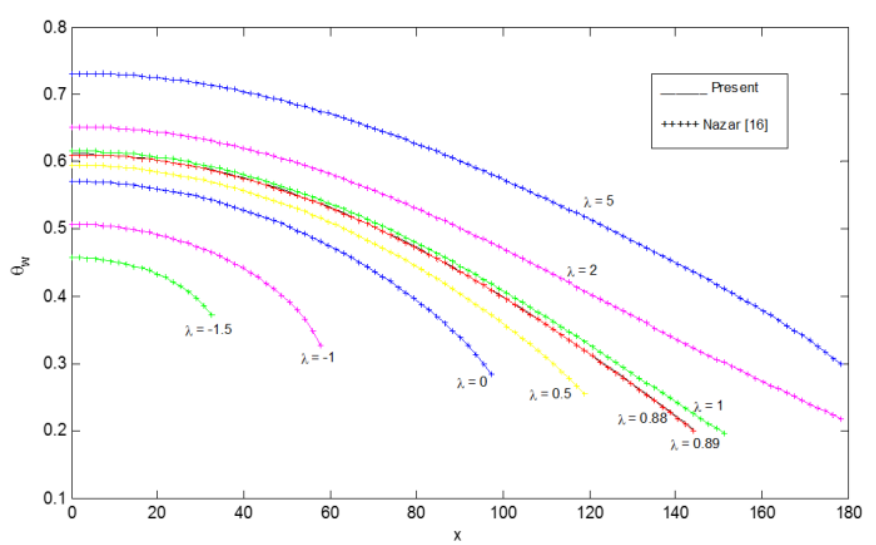

Fig.3 Comparison of the local heat transfer $\theta_{w}$ for $\mathrm{K}=0$ (Newtonian fluid), $\operatorname{Pr}=1, \phi=0$ and various of $\lambda$

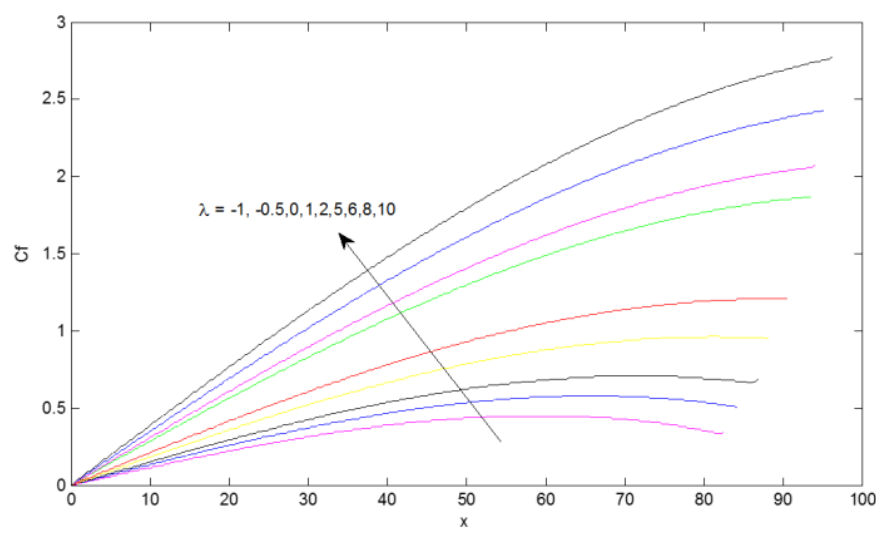

Fig.4 Local skin friction $C_{f}$ for $\mathrm{K}=1, \operatorname{Pr}=6.2, \phi=0.05$ and various of $\lambda$

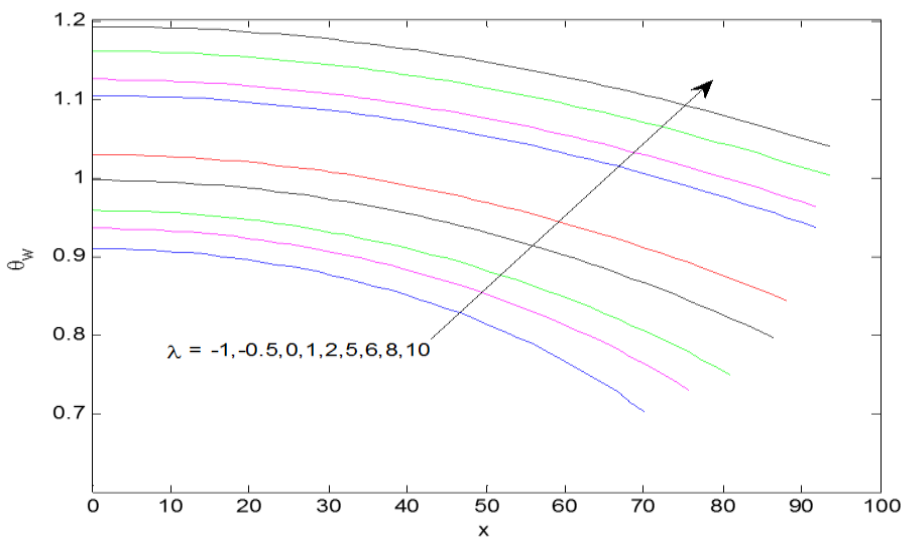

Fig.5 Local heat transfer $\theta_{w}$ for $\mathrm{K}=1, \operatorname{Pr}=6.2, \phi=0.05$ and various of $\lambda$

Further, Figures 6 and 7 show the local skin friction local skin friction $C_{f}$ and local heat transfer $\theta_{w}$ with the same value of parameter but different value of nanoparticle volume fraction, which is $\phi=0.2$. The same trend behavior is obverse as in Figures 4 and 5 . Finally, Figures 8 to 11 illustrate the velocity and temperature profiles of the cylinder against $y$ for the values of mixed convection parameter $\lambda=-1.5$ (opposing flow) and $\lambda=1$ (assisting flow) when $K=1$, $\operatorname{Pr}=6.2$ but with different values of nanoparticle volume fraction $\phi$, which are varies from $0 \leq \phi \leq 0.2$. These figures show how the nanoparticle volume fraction $\phi$ affects the fluid velocity and temperature profiles. Thus, from the graph, both velocity and temperature profiles are increased by increasing the values of nanoparticles volume fraction $\phi$ and mixed convection parameter $\lambda$.

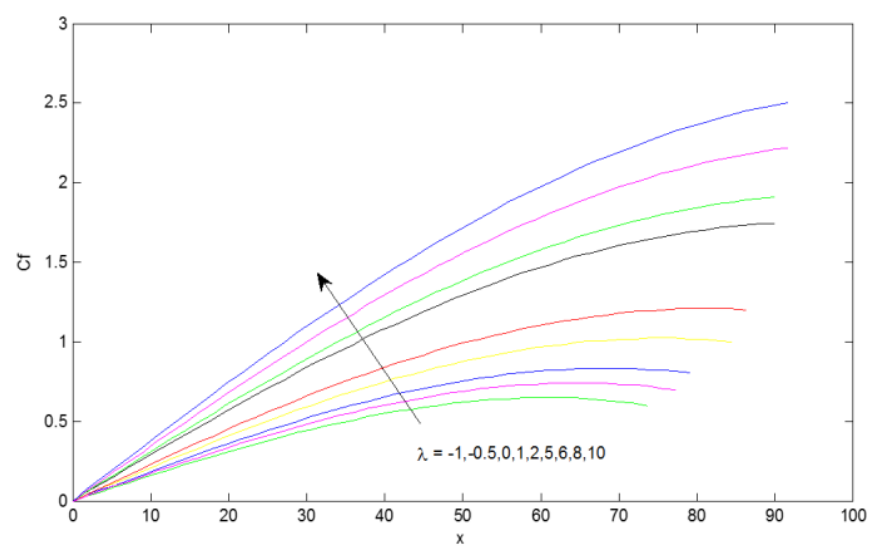

Fig.6 Local skin friction $C_{f}$ for $\mathrm{K}=1, \operatorname{Pr}=6.2, \phi=0.2$ and various of $\lambda$

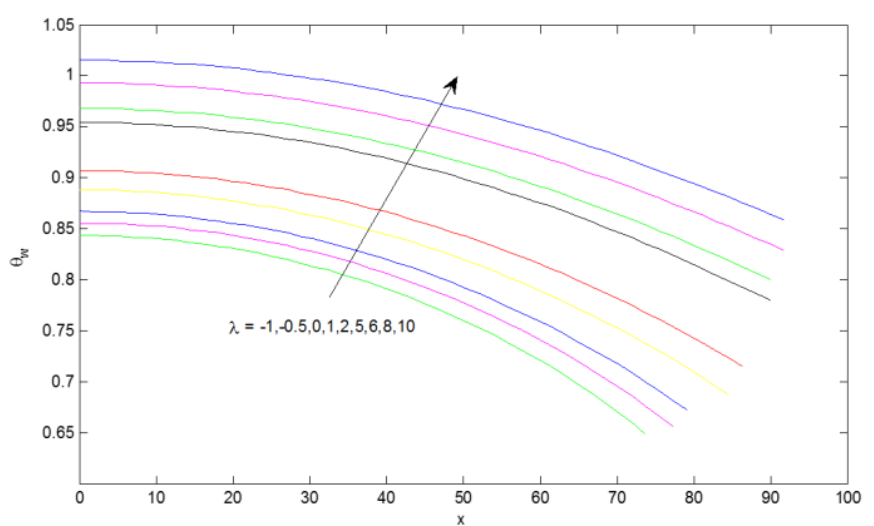

Fig.7 Local heat transfer $\theta_{w}$ for $\mathrm{K}=1, \operatorname{Pr}=6.2, \phi=0.2$ and various of $\lambda$

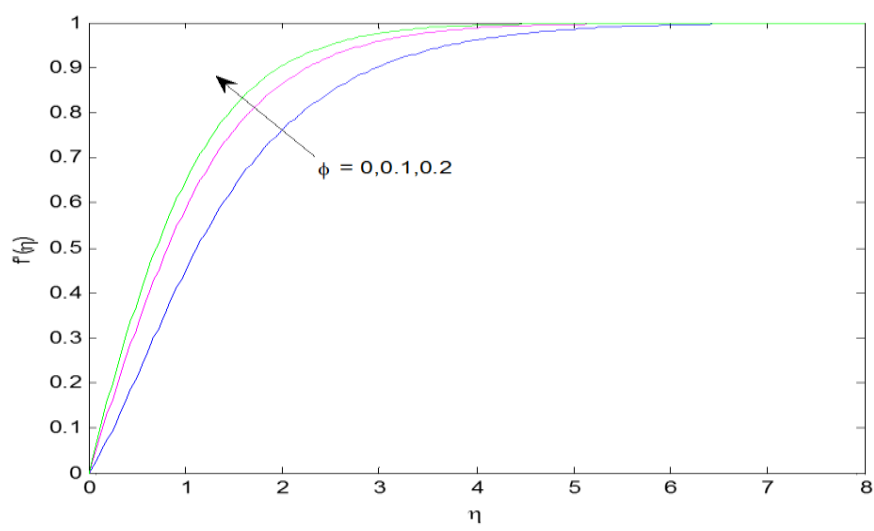

Fig.8 Velocity profiles for variety values of nanoparticle volume fraction $\phi$ for $\mathrm{K}=1, \mathrm{Pr}=6.2, \lambda=-1.5$ (opposing flow)

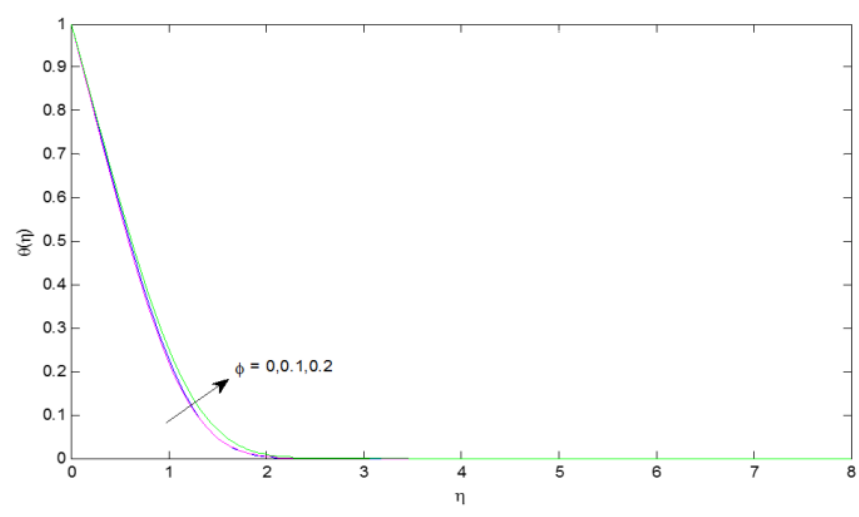

Fig.9 Temperature profiles for variety values of nanoparticle volume fraction $\phi$ for $\mathrm{K}=1, \operatorname{Pr}=6.2, \lambda=-1.5$ (opposing flow) 


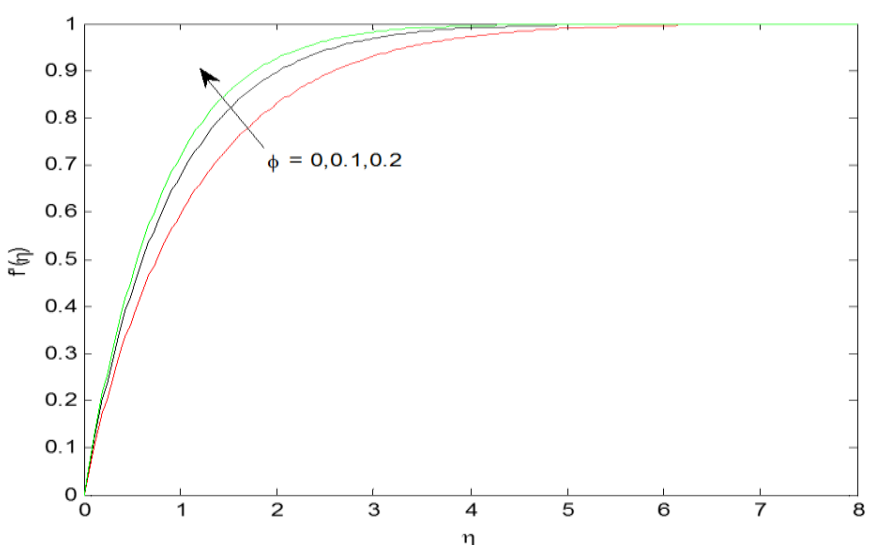

Fig.10 Velocity profiles for variety values of nanoparticle volume fraction $\phi$ for $\mathrm{K}=1, \mathrm{Pr}=6.2, \lambda=1$ (assisting flow)

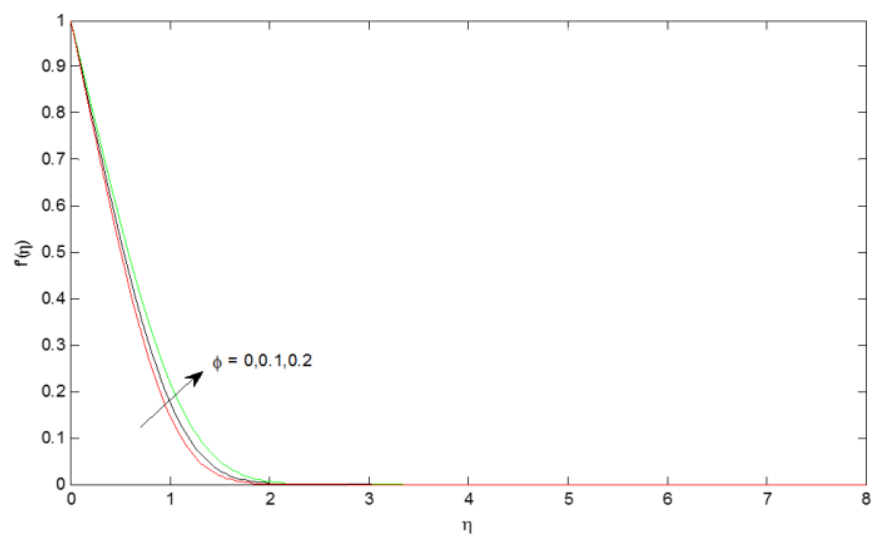

Fig.11 Temperature profiles for variety values of nanoparticle volume fraction $\phi$ for $\mathrm{K}=1, \mathrm{Pr}=6.2, \lambda=1$ (assisting flow)

\section{CONCLUSION}

Steady of mixed convection boundary layer flow of viscoelastic nanofluids past a horizontal circular cylinder with a case of constant wall temperature problem has been solved numerically using Kellerbox method. The numerical results for the skin friction, heat transfer coefficient, velocity and temperature profiles are presented for variety values of nanoparticles volume fraction, mixed convection parameter and viscoelastic parameter. The effect of velocity and temperature profiles due to the influence of nanoparticles volume fraction and mixed convection parameters are also analysed. The comparison of the results show that the present results agree very well with the previous published results reported by Nazar et al. [16] and Anwar et al. [18].

\section{ACKNOWLEDGEMENT}

The authors would like to acknowledge the Center for Research and Innovation, UniKL, Research Management Centre, UTM and Ministry of Higher Education (MOHE), STEM Grant, Malaysia for the financial support through vote numbers STRG 15095, 13H74, 4F713 and A. J091002.5600.07397 for this research.

\section{REFERENCES}

[1] S. U. Choi, "Enhancing Thermal Conductivity of Fluids with Nanoparticles," ASME Int. Mech. Eng. Congr. Expo., vol. 231, pp. 99-105, 1995.

[2] D. A. Nield and A. V. Kuznetsov, "The Cheng-Minkowycz problem for natural convective boundary-layer flow in a porous medium saturated by a nanofluid," Int. J. Heat Mass Transf., vol. 52, no. 2526, pp. 5792-5795, 2009.

[3] W. A. Khan and I. Pop, "Boundary-layer flow of a nanofluid past a stretching sheet," Int. J. Heat Mass Transf., vol. 53, no. 11-12, pp. 2477-2483, 2010.

[4] S. Ahmad and I. Pop, "Mixed convection boundary layer flow from a vertical flat plate embedded in a porous medium filled with nanofluids," Int. Commun. Heat Mass Transf., vol. 37, no. 8, pp. 987-991, 2010.

[5] A. Chamkha, R. S. R. Gorla, and K. Ghodeswar, "Non-similar Solution for Natural Convective Boundary Layer Flow Over a Sphere Embedded in a Porous Medium Saturated with a Nanofluid," Transp. Porous Media, vol. 86, no. 1, pp. 13-22, 2011.

[6] M. Qasim, I. Khan, and S. Shafie, "Heat transfer and mass diffusion in nanofluids over a moving permeable convective surface," Math. Probl. Eng., vol. 2013, 2013.

[7] A. Zaib, K. Bhattacharyya, and S. Shafie, "Unsteady boundary layer flow and heat transfer over an exponentially shrinking sheet with suction in a copper-water nanofluid," J. Cent. South Univ., vol. 22, no. 12 , pp. 4856-4863, 2015.

[8] A. Noghrehabadi, R. Pourrajab, and M. Ghalambaz, "Effect of partial slip boundary condition on the flow and heat transfer of nanofluids past stretching sheet prescribed constant wall temperature," Int. J. Therm. Sci., vol. 54, pp. 253-261, 2012.

[9] Hayat, T., Waqas, M., Sabir Ali Shehzad, Alsaedi, A., "International Journal of Numerical Methods for Heat \& Fluid Flow Article information :," Int. J. Numer. Methods Heat Fluid FLow, vol. 26, no. 1, pp. 214-234, 2016.

[10] N. A. Rawi, M. R. Ilias, Z. M. Isa, and S. Shafie, "G-Jitter induced mixed convection flow and heat transfer of micropolar nanofluids flow over an inclined stretching sheet," AIP Conf. Proc., vol. 1775, 2016.

[11] F. Mabood, W. a. Khan, and M. M. Yovanovich, "Forced Convection of Nanofluid Flow across Horizontal Circular Cylinder with Convective Boundary Condition," J. Mol. Liq., vol. 222, pp. 172-180, 2016.

[12] S. Sarkar, S. Ganguly, G. Biswas, and P. Saha, "Effect of cylinder rotation during mixed convective flow of nanofluids past a circular cylinder," Comput. Fluids, vol. 127, pp. 47-64, 2016.

[13] A. E. M. Bouchoucha and R. Bessaïh, "Natural convection in a square cavity filled with nanofluids," Fluid Dyn. Mater. Process., vol. 11, no. 3, pp. 279-300, 2015.

[14] R. K. Tiwari and M. K. Das, "Heat transfer augmentation in a twosided lid-driven differentially heated square cavity utilizing nanofluids," Int. J. Heat Mass Transf., vol. 50, no. 9-10, pp. 20022018, 2007.

[15] J. H. Merkin, "Mixed convection from a horizontal circular cylinder," Int. J. Heat Mass Transf., vol. 20, no. 1, pp. 73-77, 1977.

[16] R. Nazar, N. Amin, and I. Pop, "Mixed convection boundary-layer flow from a horizontal circular cylinder with a constant surface heat flux," Heat Mass Transf., vol. 40, no. 3-4, pp. 219-227, 2004.

[17] Y. Lin, L. Zheng, and X. Zhang, "Radiation effects on Marangoni convection flow and heat transfer in pseudo-plastic non-Newtonian nanofluids with variable thermal conductivity," Int. J. Heat Mass Transf., vol. 77, pp. 708-716, 2014.

[18] I. Anwar, N. Amin, and I. Pop, "Mixed convection boundary layer flow of a viscoelastic fluid over a horizontal circular cylinder," Int. J. Non. Linear. Mech., vol. 43, no. 9, pp. 814-821, 2008. 